\title{
Synthesis and characterisation of Fe(III) and Co(III) complexes of thiazole- containing thiosemicarbazone ligands
}

\author{
Robert J. Lavericka, Anthony B. Cartera, Harry A. Kleina, Anthony J. Fitzpatrick ${ }^{\mathrm{b}}$, Tony D. Keene ${ }^{\mathrm{b}}$, Grace \\ G. Morgan ${ }^{\mathrm{b}}$, Jonathan A. Kitchen ${ }^{\mathrm{a} *}$
}

aChemistry, University of Southampton, Southampton, S017 1BJ, UK.

bSchool of Chemistry, University College Dublin, Dublin 4, Ireland.

\section{ABSTRACT}

Three thiosemicarbazone-based ligands and corresponding Fe(III) and Co(III) coordination complexes have been synthesised and fully characterised. Crystallographic analysis of all ligands and complexes revealed interesting hydrogen bonding and $\pi$-stacking packing arrangements. Magnetic susceptibility and EPR measurements indicate that the Fe(III) complexes are in the low spin state from $4-300$ K. The complexes exhibit significant solution stability (based on UV/vis and NMR data) and the potential for further functionalisation. Such stability gives solution processability and is ideal for the development of advanced supramolecular materials.

\section{Introduction}

The development of metallosupramolecular assemblies has attracted the interest of scientists for many years and there is now a drive towards developing functional assemblies for supramolecular materials based applications. ${ }^{1-4}$ By careful choice of the metal ion a range of applications can be targeted including luminescent systems, catalysts, and magnetically interesting systems. ${ }^{5-8.9-11}$ Thiosemicarbazonebased ligands have been used to generate interesting complexes with a range of potential applications, however one area that appeals to us is their use in Fe(III) spincrossover (SCO) systems. 12-17 Most examples of thiosemicarbazone-based SCO active complexes use simple thiosemicarbazides and salicylaldehyde (SA) derivatives (e.g. Hthsa, Hth5Clsa and Hth5Brsa, Fig. 1). To the best of our knowledge, and despite the large numbers of heterocyclic aldehydes and ketones now available, there have been few studies that have used carbonyl compounds other than SA for investigation into magnetically interesting Fe(III) complexes. Additionally, for SCO-based systems to be useful in areas of nano-technology, solution processability would be advantageous as it allows for simple immobilisation into/onto substrates. Many of the magnetically interesting systems developed to date are significantly labile in solution and undergo ligand displacement giving SCO inactive compounds. ${ }^{18-22}$ Therefore developing new ligand systems that can afford favourable coordination environments for SCO and solution stability represents a major challenge in SCO research. Furthermore, ligands that have the ability to be further functionalized (through substituent addition) to allow for targeted immobilization is also desirable, therefore synthetic simplicity and modular synthesis is highly desirable. Thiosemicarbazone-based ligands are ideal for such a purpose as they are readily and reliably constructed from simple precursors allowing introduction of a large range of substituents. ${ }^{23-32}$ Here we report the synthesis and characterization of three thiazole containing

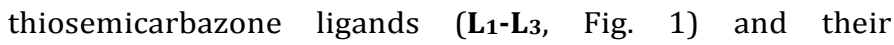
complexation with $\mathrm{Fe}(\mathrm{III})$ and $\mathrm{Co}(\mathrm{III})$. The magnetic properties of the Fe(III) systems are reported, whilst the lowspin Co(III) systems are reported as a diamagnetic system to the study of solution behavior by NMR techniques. L1 has previously been synthesised by Raposo et al.33 and used as part of a study for the recognition of anions. $\mathbf{L}_{\mathbf{3}}$ has previously been synthesised by Venkatraman et al. ${ }^{34}$ (as a different polymorph) and used in complexation studies with $\mathrm{Ni}, \mathrm{Cd}, \mathrm{Sn}$, and Hg. ${ }^{35-38}$ However, neither of these studies investigated iron or cobalt complexes for use in supramolecular materials.

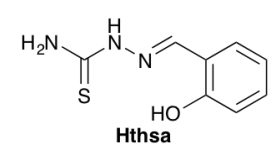<smiles>NC(=S)N/N=C/c1cc(Cl)ccc1O</smiles><smiles>NC(=S)N/N=C/c1cc(Br)ccc1O</smiles>

Fig. 1 Thiosemicarbazone ligands known to induce SCO in Fe(III) [Hthsa, Hth5Clsa, Hth5Brsa] (left) and ligands used in this study (right).

\section{Experimental}

\subsection{General}

All reagents were purchased from commercial sources and used without further purification. ${ }^{1} \mathrm{H}$, and ${ }^{13} \mathrm{C}\left\{{ }^{1} \mathrm{H}\right\}$ NMR spectra were recorded on a Bruker DPX400 NMR spectrometer at $300 \mathrm{~K}$. Chemical shifts are reported in parts per million and referenced to the residual solvent peak $\left(d_{6}-D M S O:{ }^{1} \mathrm{H} \delta 2.50 \mathrm{ppm},{ }^{13} \mathrm{C} \delta 39.52\right.$ ppm). Coupling constants (J) are reported in Hertz (Hz). Standard 
conventions indicating multiplicity were used: $\mathrm{m}=$ multiplet, $\mathrm{t}=$ triplet, $\mathrm{d}=$ doublet, $\mathrm{s}=$ singlet, $\mathrm{dd}[\mathrm{appt}]=$ doublet of doublets which appears as a triplet. Infrared spectra were recorded using a ThermoScientific Nicolet iS10 FTIR spectrometer between 600 and $4000 \mathrm{~cm}^{-1}$. Mass spectrometry samples were analysed using a MaXis (Bruker Daltonics, Bremen, Germany) mass spectrometer equipped with a Time of Flight (TOF) analyser. Samples were introduced to the mass spectrometer via a Dionex Ultimate 3000 autosampler and uHPLC pump [Gradient $20 \%$ acetonitrile $(0.2 \%$ formic acid) to $100 \%$ acetonitrile $(0.2 \%$ formic acid) in five minutes at $0.6 \mathrm{~mL} \mathrm{~min}^{-1}$. Column: Acquity UPLC BEH C18 (Waters) 1.7 micron $50 \times 2.1 \mathrm{~mm}$ ]. High-resolution mass spectra were recorded using positive/negative ion electrospray ionisation. Single-crystal X-ray diffraction data was collected at $100 \mathrm{~K}$ on a Rigaku AFC12 goniometer equipped with an enhanced sensitivity (HG) Saturn 724+ detector mounted at the window of an FR-E+ Superbright Mo-K $\alpha$ rotating anode generator $(\lambda=0.71075 \AA)$ with HF or VHF varimax optics. ${ }^{39}$ Unit cell parameters were refined against all data and an empirical absorption correction applied in either CrystalClear ${ }^{40}$ or CrysalisPro. ${ }^{41}$ All structures were solved by direct methods using SHELXS-201342 and refined on $F_{O}^{2}$ by SHELXL-201342 using Olex2.43 The crystallographic data are summarised below. CCDC entries 1498845 - 1498852 contain the crystallographic data for the structures reported in this article.

UV-Vis absorption spectra were recorded on a PerkinElmer Lambda 265 Spectrophotometer between 200 and $900 \mathrm{~nm}$. Variable temperature magnetic susceptibility for crystalline samples of all three $\mathrm{Fe}(\mathrm{III})$ complexes were recorded on a Quantum Design MPMS ${ }^{\circledR}$ XL-7 SQUID magnetometer at $0.1 \mathrm{~T}$. Magnetic susceptibility was recorded in the range of 400 to $10 \mathrm{~K}$ at $3 \mathrm{~K} / \mathrm{min}$ temperature scan rate. EPR data was collected between 283 and $373 \mathrm{~K}$ on a polycrystalline sample using a Magnettech mS200 X-band EPR working at $9.381 \mathrm{GHz}$ with magnetic field centred at $300 \mathrm{mT}$ and a field sweep of $400 \mathrm{mT}$. Modulation amplitude of $0.5 \mathrm{mT}$ was used in conjunction with a microwave power of $0.1 \mathrm{~mW}$ and a gain of 10 .

\subsection{Ligand Synthesis}

2.2.1 Synthesis of $\boldsymbol{L}_{1}$ : $\quad$ To a stirred solution of 4phenylthiosemicarbazide (200 $\mathrm{mg}, 1.20 \mathrm{mmol}$ ) in methanol (30 $\mathrm{mL})$, 2-thiazolecarboxaldehyde $(105 \mu \mathrm{L}, 1.20 \mathrm{mmol})$ was added. The light yellow solution was left to stir for 24 hours. The resulting dark yellow solution was concentrated in vacuo resulting in the precipitation of a yellow solid. This was filtered and then washed with diethyl ether $(2 \times 25 \mathrm{~mL})$, the resulting yellow solid was crystallized from methanol $(20 \mathrm{~mL})$ resulting in a light yellow crystalline solid suitable for single crystal X-ray diffraction. Yield: $181 \mathrm{mg}$ (69\%); HRMS ESI+ m/z: 285.0234 [L1 + $\mathrm{Na}]^{+}, \mathrm{C}_{11} \mathrm{H}_{10} \mathrm{~N}_{4} \mathrm{~S}_{2} \mathrm{Na}$ requires: 285.0239; IR: $v=3322,3297,3054$, $1634,1598,1279,1176,1166,1013 \mathrm{~cm}^{-1} ; \lambda_{\max }(\mathrm{MeOH})=343 \mathrm{~nm}, \varepsilon$ $=21,500 \mathrm{Lmol}^{-1} \mathrm{~cm}^{-1} ;{ }^{1} \mathrm{H} \mathrm{NMR}(400 \mathrm{MHz}$, DMSO-d $): \delta 12.09(\mathrm{~s}, 1 \mathrm{H}$, $\mathrm{N} H), 10.10(\mathrm{~s}, 1 \mathrm{H}, \mathrm{N} H), 8.39(\mathrm{~s}, 1 \mathrm{H}, \operatorname{Im} H), 7.96(\mathrm{~d}, J=3.2 \mathrm{~Hz}, 1 \mathrm{H}$, ThiaH), 7.86 (d, $J=3.2 \mathrm{~Hz}, 1 \mathrm{H}$, Thia $H$ ), $7.56-7.53$ (m, 2H, $\mathrm{Ph} H$ ), 7.41-7.35 (m, 2H, PhH), $7.23(\mathrm{~m}, 1 \mathrm{H}, \mathrm{Ph} H) .{ }^{13} \mathrm{C}$ NMR $(101 \mathrm{MHz}$, DMSO-d6): 122.29, 125.59, 125.86, 128.16, 137.29, 138.88, $144.00,163.56,176.28$. Yellow rod-like crystals $(0.35 \times 0.08 \times$ $0.07 \mathrm{~mm}$ ) of $\mathbf{L}_{\mathbf{1}}$ were obtained from hot recrystallisation from methanol. Crystal Data for $\mathrm{C}_{11} \mathrm{H}_{10} \mathrm{~N}_{4} \mathrm{~S}_{2}(M=262.35 \mathrm{~g} / \mathrm{mol})$ : monoclinic, space group $P 21 / c$ (no. 14), $a=13.5767(10) \AA, b=$ $5.3420(4) \AA, c=\quad 17.5065(11) \AA, \beta=\quad 110.324(6)^{\circ}, V=$ $1190.64(15) \AA^{3}, Z=4, T=100(2) \mathrm{K}, \mu\left(\mathrm{MoK}_{\alpha}\right)=0.428 \mathrm{~mm}$
1, Dcalc $=1.464 \mathrm{~g} / \mathrm{cm}^{3}, 8078$ reflections measured $\left(4.962^{\circ} \leq 2 \Theta \leq\right.$ $\left.55.082^{\circ}\right), 2728$ unique $\left(R_{\text {int }}=0.0273, R_{\text {sigma }}=0.0221\right)$ which were used in all calculations. The final $R_{1}$ was 0.0276 (I > $2 \sigma(\mathrm{I})$ ) and $w R_{2}$ was 0.0715 (all data).

2.2.2 Synthesis of $\boldsymbol{L}_{2}$ : To a stirred solution of 4phenylthiosemicarbazide (200 mg, $1.20 \mathrm{mmol})$ in methanol (30 $\mathrm{mL})$, 4-methyl-2-thiazolecarboxaldehyde (129 $\mu \mathrm{L}, 1.20 \mathrm{mmol}$ ) was added. The solution was stirred for 24 hours. The resulting dark yellow solution was concentrated in vacuo, leaving a dark yellow oil. This was triturated in diethyl either $(20 \mathrm{~mL})$ and decanted before being recrystallized from methanol $(20 \mathrm{~mL})$ resulting in a dark yellow crystalline solid. Yield: $133 \mathrm{mg}$ (48\%); HRMS ESI+ m/z: $299.0395\left[\mathbf{L}_{2}+\mathrm{Na}\right]^{+}, \mathrm{C}_{12} \mathrm{H}_{12} \mathrm{~N}_{4} \mathrm{~S}_{2} \mathrm{Na}$ requires: 299.0396; IR: $v=3317,3107,3054,1596,1538,1236,1176$, $1165,1031 \mathrm{~cm}^{-1} ; \lambda_{\max }(\mathrm{MeOH})=349 \mathrm{~nm}, \varepsilon=22,600 \mathrm{Lmol}^{-1} \mathrm{~cm}^{-1} ;{ }^{1} \mathrm{H}$ NMR (300 MHz, DMSO-d $) \delta 12.05(\mathrm{~s}, 1 \mathrm{H}, \mathrm{NH}), 10.04(\mathrm{~s}, 1 \mathrm{H}, \mathrm{NH})$, $8.33(\mathrm{~s}, 1 \mathrm{H}, \mathrm{Im} H), 7.54(\mathrm{~m}, 2 \mathrm{H}, \mathrm{Ph} H), 7.40-7.34(\mathrm{~m}, 3 \mathrm{H}, \mathrm{Ph} H$ \& ThiaH), 7.24-7.19 (m, 1H, $\mathrm{Ph} H$ ), 2.39 (s, 3H, Methyl $H$ ). ${ }^{13} \mathrm{C}$ NMR (101 MHz, DMSO-d6): 17.17, 117.17, 126.00. 126.29, 128.61, $137.83,139.36,153.85,163.07,176.66$. Orange rod-like crystals $(0.27 \times 0.05 \times 0.04 \mathrm{~mm})$ of $\mathbf{L}_{2}$ were obtained from hot recrystallisation from methanol. Crystal Data for $\mathrm{C}_{12} \mathrm{H}_{12} \mathrm{~N}_{4} \mathrm{~S}_{2}(M=276.38 \mathrm{~g} / \mathrm{mol})$ : orthorhombic, space group $P 2{ }_{1} 2{ }_{1} 2_{1}$ (no. 19), $a=5.004(5) \AA, b=15.83(2) \AA, c=16.73(2) \AA, V=$ $1325(3) \AA^{3}, Z=4, T=373.3 \mathrm{~K}, \mu\left(\mathrm{MoK}_{\alpha}\right)=0.389 \mathrm{~mm}^{-1}$, Dcalc $=$ $1.385 \mathrm{~g} / \mathrm{cm}^{3}, 4836$ reflections measured $\left(5.694^{\circ} \leq 2 \Theta \leq 50.988^{\circ}\right)$, 2427 unique $\left(R_{\text {int }}=0.0305\right.$, $\left.R_{\text {sigma }}=0.0489\right)$ which were used in all calculations. The final $R_{1}$ was 0.0440 (I $>2 \sigma(\mathrm{I})$ ) and $w R_{2}$ was 0.0767 (all data).

2.2.3 Synthesis of $\boldsymbol{L}_{3}$ : To a stirred solution of 4phenylthiosemicarbazide $(100 \mathrm{mg}, 0.6 \mathrm{mmol})$ in ethanol $(10 \mathrm{~mL})$, 2 -acetylthiazole $(70 \mu \mathrm{L}, 0.6 \mathrm{mmol})$ was added. The solution was refluxed for 3 hours. The solution was concentrated resulting in the precipitation of a yellow crystalline solid that was filtered, washed with diethyl ether ( $2 \times 25 \mathrm{~mL})$ and dried in vacuo. Yield $84 \mathrm{mg}, 51 \%$. HRMS ESI+ m/z: $277.0574\left[\mathbf{L}_{3}+\mathrm{H}^{+}, \mathrm{C}_{12} \mathrm{H}_{13} \mathrm{~N}_{4} \mathrm{~S}_{2}\right.$ requires: 277.0576; IR $v=3238,3112,3037,1651,1479,1371$, $1046 \mathrm{~cm}^{-1} ; \lambda_{\max }(\mathrm{MeOH})=339 \mathrm{~nm}, \varepsilon=20,500 \mathrm{Lmol}^{-1} \mathrm{~cm}^{-1} ;{ }^{1} \mathrm{H} \mathrm{NMR}$ (400 MHz, DMSO- $\left.d_{6}\right) \delta 11.01(\mathrm{~s}, 1 \mathrm{H}, \mathrm{N} H), 9.92(\mathrm{~s}, 1 \mathrm{H}, \mathrm{N} H), 7.91(\mathrm{~d}$, $J=3.2 \mathrm{~Hz}, 1 \mathrm{H}$, Thia $H$ ), 7.82 (d, $J=3.2 \mathrm{~Hz}, 1 \mathrm{H}$, Thia $H$ ), $7.63-7.59$ $(\mathrm{m}, 2 \mathrm{H}, \mathrm{Ph} H$ ), 7.38 (dd[appt], $J=8.1 \mathrm{~Hz}, 2 \mathrm{H}, \mathrm{Ph} H), 7.23-7.19$ (m, $1 \mathrm{H}, \mathrm{Ph} H)$, MethylH signal masked by solvent peaks. ${ }^{13} \mathrm{C}$ NMR $(101$ MHz, DMSO- $\left.d_{6}\right) \delta 14.44,123.22,125.53,125.89,128.72,139.36$, $143.82,145.57,167.25,177.30$. Yellow plate like crystals $(0.4 \times$ $0.2 \times 0.09 \mathrm{~mm}$ ) of $\mathbf{L}_{3}$ were grown from the slow evaporation of methanol. Crystal Data for $\mathrm{C}_{12} \mathrm{H}_{12} \mathrm{~N}_{4} \mathrm{~S}_{2}(M=276.37 \mathrm{~g} / \mathrm{mol})$ : triclinic, space group $P-1$ (no. 2), $a=8.211 \AA$, $b=8.581 \AA$, $c=$ $9.241 \AA, \alpha=85.44^{\circ}, \beta=79.51^{\circ}, \gamma=80.63^{\circ}, V=630.8 \AA^{3}, Z=2, T=$ $100 \mathrm{~K}, \mu\left(\mathrm{MoK}_{\alpha}\right)=0.408 \mathrm{~mm}^{-1}$, Dcalc $=1.455 \mathrm{~g} / \mathrm{cm}^{3}, \quad 5325$ reflections measured $\left(6.192^{\circ} \leq 2 \Theta \leq 50.99^{\circ}\right), 2326$ unique $(R$ int $=$ $0.0144, R_{\text {sigma }}=0.0155$ ) which were used in all calculations. The final $R_{1}$ was $0.0296\left(\mathrm{I}>2 \sigma(\mathrm{I})\right.$ ) and $w R_{2}$ was 0.0812 (all data).

\subsection{Complex synthesis}

Unless stated otherwise, to a stirred solution of metal salt $\mathrm{Fe}\left(\mathrm{NO}_{3}\right)_{3} \cdot 9 \mathrm{H}_{2} \mathrm{O}(24.0 \mathrm{mg}, 0.06 \mathrm{mmol})$ or $\mathrm{Co}\left(\mathrm{BF}_{4}\right)_{2} \cdot 6 \mathrm{H}_{2} \mathrm{O}(20.2 \mathrm{mg}$, $0.06 \mathrm{mmol})$ ) in methanol $(20 \mathrm{~mL})$, ligand $\left(\mathbf{L}_{1}, \mathbf{L}_{2}\right.$ or $\left.\mathbf{L}_{3}\right)(0.12$ mmol) was added. The resulting solution was stirred vigorously 
for an hour at room temperature, before being subjected to vapour diffusion of diethyl either, which resulted in very dark crystals:

2.3.1 $\left[\mathrm{Fe}\left(\mathrm{L}_{1}\right)_{2}\right]\left(\mathrm{NO}_{3}\right)$ - Yield: $30.4 \mathrm{mg}(80 \%) ; \mathrm{HRMS}$ ESI+ m/z: $577.9890\left[\mathrm{Fe}\left(\mathbf{L}_{1}\right)_{2}-\mathrm{NO}_{3}\right]^{+}, \mathrm{C}_{22} \mathrm{H}_{18} \mathrm{~N}_{8} \mathrm{~S}_{4} \mathrm{Fe}$ requires: 577.9881. IR: $v=3179,3126,3028,3008,1594,1542,1309,1283,1193,1181$ $(\mathrm{NN}), 1001 \mathrm{~cm}^{-1}$. UV/vis (MeOH) $\lambda_{\max }=402 \mathrm{~nm}, \varepsilon=43,650 \mathrm{~L} \mathrm{~mol}^{-1}$ $\mathrm{cm}^{-1}$. Dark orange, plate like crystals of $\left[\mathrm{Fe}\left(\mathbf{L}_{1}\right)_{2}\right]\left(\mathrm{NO}_{3}\right) \cdot \mathrm{H}_{2} \mathrm{O}(0.48 \times$ $0.09 \times 0.04 \mathrm{~mm}$ ) were grown by diffusion of diethyl ether into the reaction solution. Crystal Data for $\mathrm{C}_{22} \mathrm{H}_{20} \mathrm{FeN}_{9} \mathrm{O}_{4} \mathrm{~S}_{4}(M=658.56$ g/mol): monoclinic, space group $P 21 / n$ (no. 14), $a=$ 12.0840 (8) $\AA, b=\quad 14.3302(10) \AA, c=\quad 15.8785(11) \AA, \beta=$ $105.4500(10)^{\circ}, V=2650.3(3) \AA^{3}, Z=4, T=100.15 \mathrm{~K}, \mu\left(\mathrm{MoK}_{\alpha}\right)=$ $0.934 \mathrm{~mm}^{-1}$, Dcalc $=1.651 \mathrm{~g} / \mathrm{cm}^{3}, 15780$ reflections measured $\left(6.036^{\circ} \leq 2 \Theta \leq 50.998^{\circ}\right), 4914$ unique $\left(R_{\text {int }}=0.0303, \mathrm{R}_{\text {sigma }}=\right.$ $0.0266)$ which were used in all calculations. The final $R_{1}$ was $0.0347(\mathrm{I}>2 \sigma(\mathrm{I}))$ and $w R_{2}$ was 0.0950 (all data).

2.3.2 $\left[\mathrm{Fe}\left(\mathrm{L}_{2}\right)_{2}\right]\left(\mathrm{NO}_{3}\right)$ - Yield: $30.1 \mathrm{mg}(76 \%)$; HRMS ESI+ m/z: $606.0204\left[\mathrm{Fe}\left(\mathbf{L}_{2}\right)_{2}-\mathrm{NO}_{3}\right]^{+}, \mathrm{C}_{24} \mathrm{H}_{22} \mathrm{~N}_{8} \mathrm{~S}_{4} \mathrm{Fe}$ requires: 606.0194. IR: $v=3026,2953,2920,1604,1592,1335,1291,1207,1182$, 982.cm-1. UV/vis (MeOH) $\lambda_{\max }=406 \mathrm{~nm}, \varepsilon=43,240 \mathrm{~L} \mathrm{~mol}^{-1} \mathrm{~cm}^{-1}$. Dark orange, plate like crystals of $\left[\mathrm{Fe}\left(\mathbf{L}_{2}\right)_{2}\right]\left(\mathrm{NO}_{3}\right) \cdot \mathrm{H}_{2} \mathrm{O}(0.31 \times 0.10$ $\times 0.09 \mathrm{~mm}$ ) were grown by diffusion of diethyl ether into the reaction solution. Crystal Data for $\mathrm{C}_{24} \mathrm{H}_{24} \mathrm{FeN}_{9} \mathrm{O}_{4} \mathrm{~S}_{4}(M=686.61$ g/mol): monoclinic, space group $P 21 / c$ (no. 14), $a=$ 9.0741(5) $\AA, b=\quad 14.1019(10) \AA, c=\quad 22.4772(16) \AA, \beta=$ 98.271(2) ${ }^{\circ}, V=2846.3(3) \AA^{3}, Z=4, T=100.15 \mathrm{~K}, \mu\left(\mathrm{MoK}_{\alpha}\right)=0.873$ $\mathrm{mm}^{-1}$, Dcalc $=1.602 \mathrm{~g} / \mathrm{cm}^{3}, 16157$ reflections measured $\left(5.406^{\circ} \leq\right.$ $\left.2 \Theta \leq 50.998^{\circ}\right), 5261$ unique $\left(R_{\text {int }}=0.0724, \mathrm{R}_{\text {sigma }}=0.0750\right)$ which were used in all calculations. The final $R_{1}$ was 0.0528 (I $>2 \sigma(\mathrm{I})$ ) and $w R_{2}$ was 0.1317 (all data).

2.3.3 $\left[\mathrm{Fe}\left(\mathrm{L}_{3}\right)_{2}\right]\left(\mathrm{NO}_{3}\right)$ - Yield: $30.1 \mathrm{mg}$ (76\%); HRMS ESI+ m/z: 606.0189 [ $\left.\mathrm{Fe}\left(\mathbf{L}_{3}\right)_{2}-\mathrm{NO}_{3}\right]^{+}, \mathrm{C}_{24} \mathrm{H}_{22} \mathrm{~N}_{8} \mathrm{~S}_{4} \mathrm{Fe}$ requires: 606.0194. IR: $v=3223,3076,1596,1364,1296,1244,1038,690 \mathrm{~cm}^{-1} \mathrm{UV} / \mathrm{vis}$ $(\mathrm{MeOH}) \lambda_{\max }=397 \mathrm{~nm}, \varepsilon=43,120 \mathrm{~L} \mathrm{~mol}^{-1} \mathrm{~cm}^{-1}$. Dark orange, plate like crystals of $\left[\mathrm{Fe}\left(\mathbf{L}_{3}\right)_{2}\right]\left(\mathrm{NO}_{3}\right) \cdot 3 / 4 \mathrm{H}_{2} \mathrm{O}(0.19 \times 0.12 \times 0.05 \mathrm{~mm})$ were grown by diffusion of diethyl ether into the reaction solution. Crystal Data for $\mathrm{C}_{24} \mathrm{H}_{23.5} \mathrm{FeN}_{9} \mathrm{O}_{3.75} \mathrm{~S}_{4}(M=682.11 \mathrm{~g} / \mathrm{mol})$ : orthorhombic, space group Pna21 (no. 33), $a=14.0509$ (8) $\AA, b=$ 22.7382(13) $\AA, c=\quad 8.6415(5) \AA ⿻, V=2760.9(3) \AA^{3}, Z=\quad 4, T=$ $100.15 \mathrm{~K}, \mu\left(\mathrm{MoK}_{\alpha}\right)=0.899 \mathrm{~mm}^{-1}$, Dcalc $=1.641 \mathrm{~g} / \mathrm{cm}^{3}, 11487$ reflections measured $\left(4.714^{\circ} \leq 2 \Theta \leq 49.99^{\circ}\right), 4787$ unique $\left(R_{\text {int }}=\right.$ $0.0483, R_{\text {sigma }}=0.0829$ ) which were used in all calculations. The final $R_{1}$ was 0.0687 ( $\mathrm{I}>2 \sigma(\mathrm{I})$ ) and $w R_{2}$ was 0.1807 (all data), twinned data refinement scales for twin law -h, k, -l: 0.67(5) 0.33(5).

2.3.4 $\left[\mathrm{Co}\left(L_{1}\right)_{2}\right]\left(B F_{4}\right)$ - Yield: $27.0 \mathrm{mg}(68 \%) ; \mathrm{MS}$ ESI+ m/z: $580.9855\left[\mathrm{Co}\left(\mathrm{L}_{1}\right)_{2}-\mathrm{BF}_{4}\right]^{+}, \mathrm{C}_{22} \mathrm{H}_{18} \mathrm{CoN}_{8} \mathrm{~S}_{4}$ requires: 580.9864; IR: $v=3328,3137,3078,3038,2973,1593,1526,1417,13981314$, 1293, 1191, 1149, $1000 \mathrm{~cm}^{-1} ;{ }^{1} \mathrm{H}$ NMR (400 MHz, DMSO-d $): \delta$ $10.60(\mathrm{~s}, 2 \mathrm{H}, \mathrm{NH}), 9.02(\mathrm{~s}, 2 \mathrm{H}, \operatorname{Im} H), 8.06(\mathrm{~d}, J=3.4 \mathrm{~Hz}, 2 \mathrm{H}$, Thia $H$ ), 7.68 (d, $J=3.4 \mathrm{~Hz}, 2 \mathrm{H}$, Thia $H$ ), 7.60 (d, $J=8.0 \mathrm{~Hz}, 4 \mathrm{H}, \mathrm{Ph} H$ ), $7.39-$ $7.34(\mathrm{~m}, 4 \mathrm{H}, \mathrm{Ph} H), 7.12(\mathrm{~m}, 2 \mathrm{H}, \mathrm{Ph} H)$. Small, dark red, plate like crystals of $\left[\mathrm{Co}\left(\mathrm{L}_{1}\right)_{2}\right]\left(\mathrm{BF}_{4}\right) \cdot 1 \frac{1}{2} \mathrm{H}_{2} \mathrm{O}(0.09 \times 0.04 \times 0.01 \mathrm{~mm})$ were grown by diffusion of diethyl ether into the reaction solution. Crystal Data for $\mathrm{C}_{22} \mathrm{H}_{19} \mathrm{BN}_{8} \mathrm{O}_{0.5} \mathrm{~F}_{4} \mathrm{~S}_{4} \mathrm{Co} \quad(M=677.43 \mathrm{~g} / \mathrm{mol})$ : monoclinic, space group $P 21 / n$ (no. 14), $a=12.120(2) \AA$, $b=$ 14.421(3) $\AA, c=\quad 15.666(3) \AA \AA, \beta=\quad 103.54(3)^{\circ}, V=$ 2662.1(10) $\AA^{3}, Z=4, T=100.15 \mathrm{~K}, \mu\left(\mathrm{MoK}_{\alpha}\right)=1.020 \mathrm{~mm}^{-1}$, Dcalc $=$ $1.690 \mathrm{~g} / \mathrm{cm}^{3}, 15530$ reflections measured $\left(4.464^{\circ} \leq 2 \Theta \leq 50.996^{\circ}\right)$, 4948 unique $\left(R_{\text {int }}=0.0427, R_{\text {sigma }}=0.0420\right)$ which were used in all calculations. The final $R_{1}$ was $0.0398\left(\mathrm{I}>2 \sigma(\mathrm{I})\right.$ ) and $w R_{2}$ was 0.1000 (all data).

2.3.5 $\left[\mathrm{Co}\left(\mathrm{L}_{2}\right)_{2}\right]\left(\mathrm{BF}_{4}\right)$ - Yield: $29.3 \mathrm{mg}$ (71\%); MS ESI+ m/z: 609.0188 [ $\left.\mathrm{Co}\left(\mathbf{L}_{\mathbf{1}}\right)_{2}-\mathrm{BF}_{4}\right]^{+}, \mathrm{C}_{24} \mathrm{H}_{22} \mathrm{CoN}_{8} \mathrm{~S}_{4}$ requires: 609.0177; IR: $v=3308,3105,3076,3038,(\mathrm{NN}), 987 ; \lambda_{\max }=402 \mathrm{~nm} ;{ }^{1} \mathrm{H} \mathrm{NMR}$ (400 MHz, DMSO-d6): $\delta 10.65$ (s, 2H, NH), 9.01 (s, 2H, ImH), 7.72 (s, 2H, Thia $H$ ), 7.58 (d, $J=8.0 \mathrm{~Hz}, 4 \mathrm{H}, \mathrm{Ph} H$ ), 7.37 (dd[appt], $J=7.7$ $\mathrm{Hz}, 4 \mathrm{H}, \mathrm{Ph} H$ ), 7.12 (t, $J=7.4 \mathrm{~Hz}, 2 \mathrm{H}, \mathrm{Ph} H$ ), 2.22 (s, 6H, Methyl $H$ ). Small, dark red, needle like crystals of $\left[\mathrm{Co}\left(\mathbf{L}_{2}\right)_{2}\right]\left(\mathrm{BF}_{4}\right) \cdot \mathrm{Et}_{2} \mathrm{O}(0.12 \times$ $0.06 \times 0.02 \mathrm{~mm}$ ) were grown by diffusion of diethyl ether into the reaction solution. Crystal Data for $\mathrm{C}_{26} \mathrm{H}_{27} \mathrm{BCoF}_{4} \mathrm{~N}_{8} \mathrm{O}_{0.5} \mathrm{~S}_{4}(M=733.53 \mathrm{~g} / \mathrm{mol})$ : monoclinic, space group $P 21 / c \quad$ (no. 14), $a=10.9533(8) \AA \AA, b=12.7844$ (9) $\AA$, $c=$ 23.1491(16) $\AA, \beta=93.5960(10)^{\circ}, V=3235.2(4) \AA^{3}, Z=4, T=$ $100.15 \mathrm{~K}, \mu\left(\mathrm{MoK}_{\alpha}\right)=0.846 \mathrm{~mm}^{-1}$, Dcalc $=1.506 \mathrm{~g} / \mathrm{cm}^{3}, 30141$ reflections measured $\left(5.288^{\circ} \leq 2 \Theta \leq 50^{\circ}\right), 5678$ unique $\left(R_{\text {int }}=\right.$ $\left.0.0575, R_{\text {sigma }}=0.0312\right)$ which were used in all calculations. The final $R_{1}$ was 0.0511 ( $>2 \sigma(\mathrm{I})$ ) and $w R_{2}$ was 0.1381 (all data).

2.3.6 $\left[\mathrm{Co}\left(\mathrm{L}_{3}\right)_{2}\right]\left(\mathrm{BF}_{4}\right)$ - To a stirred solution of metal salt $\mathrm{Co}\left(\mathrm{BF}_{4}\right)_{2} \cdot 6 \mathrm{H}_{2} \mathrm{O}(12.7 \mathrm{mg}, 0.04 \mathrm{mmol})$ ) in methanol $(20 \mathrm{~mL})$, ligand (L3) $(0.07 \mathrm{mmol})$ was added. Yield: $17.8 \mathrm{mg}(70 \%)$; MS ESI+ m/z: $609.0181\left[\mathrm{Co}\left(\mathrm{L}_{1}\right)_{2}-\mathrm{BF}_{4}\right]^{+}, \mathrm{C}_{24} \mathrm{H}_{22} \mathrm{CoN}_{8} \mathrm{~S}_{4}$ requires 609.0177; IR: $v=$ $3228,2161,1496,1431,1047,750,575 \mathrm{~cm}^{-1} ; \lambda_{\max }=402 \mathrm{~nm} ;{ }^{1} \mathrm{H}$ NMR (400 MHz, DMSO-d6): $\delta 10.74-9.90$ (bs, 2H, NH), 8.08 (d, $J=$ $3.5 \mathrm{~Hz}, 2 \mathrm{H}, \mathrm{Thia} H), 7.69-7.66(\mathrm{~m}, 6 \mathrm{H}, \mathrm{Ph} H$ \& Thia $H), 7.37$ (dd[appt], $J=7.8 \mathrm{~Hz}, 4 \mathrm{H}, \mathrm{Ph} H$ ), $7.09(\mathrm{t}, J=7.4 \mathrm{~Hz}, 2 \mathrm{H}, \mathrm{Ph} H), 2.95$ (s,
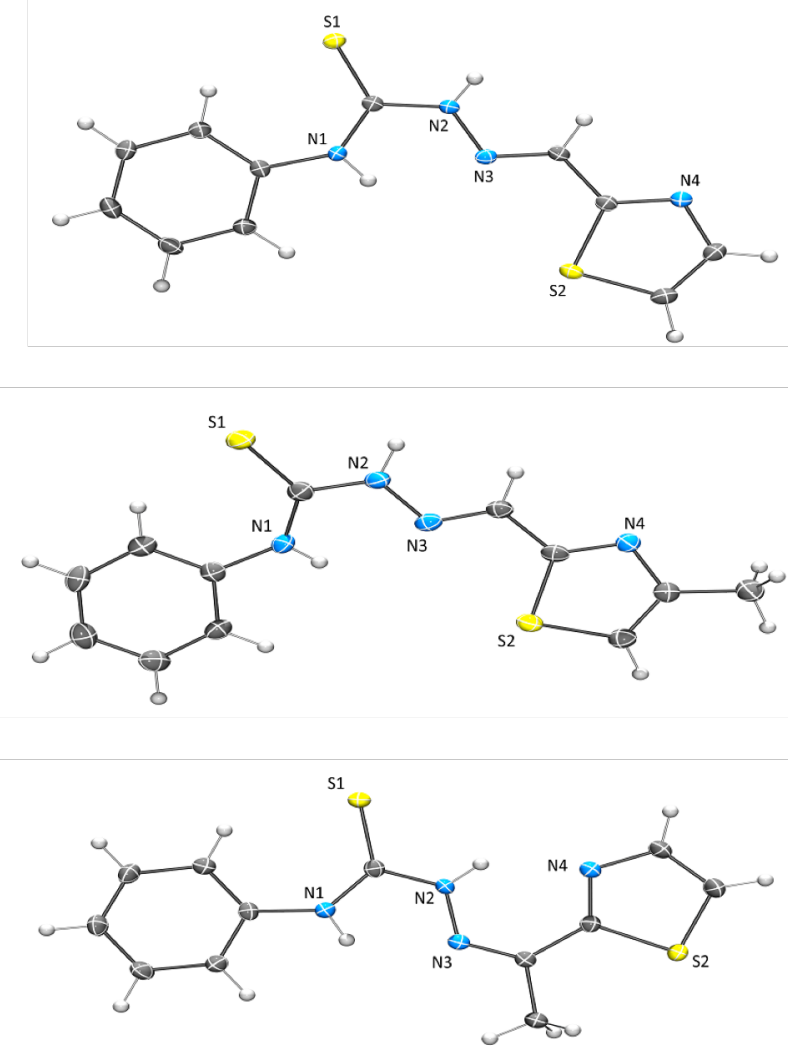

Fig. 2 Molecular structures of $\mathbf{L}_{\mathbf{1}}$ (top), $\mathbf{L}_{\mathbf{2}}$ (middle) and $\mathbf{L}_{\mathbf{3}}$ (bottom). Thermal ellipsoids shown at $50 \%$ probability. 
$6 \mathrm{H}, \mathrm{Methyl} H$ ). Despite repeated attempts, and under a variety of conditions only very poor quality dark orange block like crystals $(0.11 \times 0.10 \times 0.08 \mathrm{~mm})$ were grown by diffusion of diethyl ether into a methanol solution. The data quality was sufficiently poor that no packing interactions are discussed and the molecular structure serves to show the connectivity of the $\left[\operatorname{Co}\left(\mathbf{L}_{3}\right)_{2}\right]$ cation only. Crystal Data for $\mathrm{C}_{24} \mathrm{H}_{21} \mathrm{CoN}_{8} \mathrm{~S}_{4}(M=608.66 \mathrm{~g} / \mathrm{mol})$ : orthorhombic, space group Pbca (no. 61), $a=8.4306(2) \AA, b=$ 21.5049(6) $\AA, c=33.7844(11) \AA ̊, V=6125.1(3) \AA^{3}, Z=8, T=$ $100 \mathrm{~K}, \mu(\mathrm{MoK} \alpha)=0.860 \mathrm{~mm}^{-1}$, Dcalc $=1.320 \mathrm{~g} / \mathrm{cm}^{3}, \quad 48295$ reflections measured $\left(3.788^{\circ} \leq 2 \Theta \leq 49.998^{\circ}\right), 5383$ unique $\left(R_{\text {int }}=\right.$ $0.0609, \mathrm{R}_{\text {sigma }}=0.0380$ ) which were used in all calculations. The final $R_{1}$ was 0.0750 (I $>2 \sigma(\mathrm{I})$ ) and $w R_{2}$ was 0.2076 (all data). The structure was treated with a smtbx solvent mask in Olex2 to remove the severely disordered $\mathrm{BF}_{4}^{-}$counter anion and an interstitial water molecule.

\section{Results and Discussion}
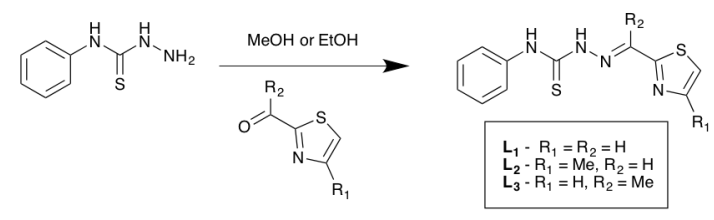

Scheme 1 General synthesis of $\mathbf{L}_{\mathbf{1}}-\mathbf{L}_{\mathbf{3}}$

\subsection{Ligand synthesis and characterisation}

Ligands $\mathbf{L}_{\mathbf{1}}$ - $\mathbf{L}_{\mathbf{3}}$ (Fig. 2) were synthesised by the same general procedure (Scheme 1) using a stoichiometric reaction between the thiazole carbonyl (2-thiazolecarboxaldehyde, 4-methyl-2thiazolecarboxaldehyde or 2-acetylthiazole) and 4phenylthiosemicarbazide in refluxing methanol or ethanol to yield L1, L2 and L3 respectively. Following the reaction, concentration of solvent resulted in pale orange/yellow solids in moderate yields (48 - 69\%). $\mathbf{L}_{\mathbf{1}}-\mathbf{L} \mathbf{3}$ were fully characterised using ${ }^{1} \mathrm{H}-\mathrm{NMR}$, ${ }^{13} \mathrm{C}$-NMR, IR, UV/vis, mass spectrometry and X-ray crystallography. All spectroscopic data was consistent with the formation of the desired thiosemicarbazone ligands (see supporting information). $\mathbf{L}_{\mathbf{1}}$ and $\mathbf{L}_{\mathbf{3}}$ match the literature data. $\mathbf{3 0 , 3 1}^{\mathbf{3}}$ Large pale yellow crystals of $\mathbf{L}_{\mathbf{1}}$ were obtained by hot recrystallisation from methanol. The ligand crystallised in the monoclinic space group $P 2_{1} / c$, with one molecule in the asymmetric unit. The ligand adopts a relatively planar structure with respect to the angles formed between the thiazole thiosemicarbazone mean-plane $\left(4.7^{\circ}\right)$ and the thiosemicarbazone - phenyl mean-plane $\left(14.3^{\circ}\right)$. The thiourea moiety adopts an anticonformation and the imine bond adopts a trans configuration (i.e. thiazole group and thiourea $\mathrm{NH}$ are trans). When examining the long range packing two perpendicular planes of ligand run though the crystal along the $\mathrm{b}$ axis (Fig. S44, ESI), this is facilitated through intermolecular hydrogen bonding $\left[\mathrm{N}(3) \cdots \mathrm{N}(1)^{\prime}=\right.$ $2.980(2) \AA$ and $\left.<\left(\mathrm{N}(3)-\mathrm{H}(3 \mathrm{X}) \cdots \mathrm{N}(1)^{\prime}\right)=164^{\circ}\right]$. Small, dark orange, rod-like crystals of $\mathbf{L}_{2}$ were obtained from hot recrystallisation from methanol and crystallised in the chiral orthorhombic space group $P 2{ }_{1} 2{ }_{1} 2_{1}$, and contained one molecule in the asymmetric unit. The ligand conformation is very similar to that of $\mathbf{L}_{\mathbf{1}}$, where it adopts a relatively flat structure with respect to the angles formed between the thiazole - thiosemicarbazone mean-plane $\left(11.4^{\circ}\right)$ and the thiosemicarbazone - phenyl mean-plane $\left(5.8^{\circ}\right)$. Again the thiourea moiety adopts an anti-conformation and the imine bond adopts a trans configuration as per $\mathbf{L}_{\mathbf{1}}$. The long range packing differs significantly to $\mathbf{L}_{\mathbf{1}}$ as the intermolecular hydrogen bonding

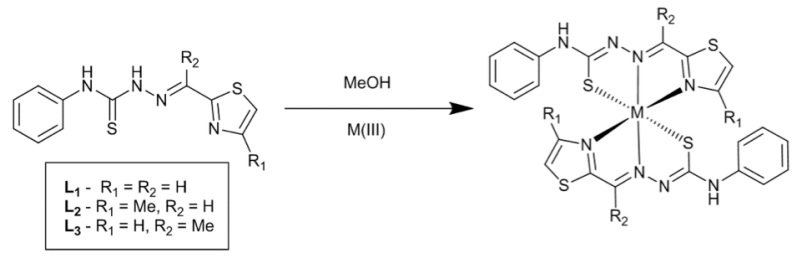

Scheme 2 Complexation of ligands $\mathbf{L}_{\mathbf{1}}-\mathbf{L}_{3}$ with Fe(III) or Co(III).

in $\mathbf{L}_{2}\left[\mathrm{~N}(3) \cdots \mathrm{N}(1)^{\prime}=3.025(5) \AA\right.$ and $\left.<\left(\mathrm{N}(3)-\mathrm{H}(3 \mathrm{X}) \cdots \mathrm{N}(1)^{\prime}\right)=158^{\circ}\right]$ results in a helical hydrogen bonded $1 \mathrm{D}$ chain along the crystallographic a-axis. Small pale yellow crystals of $\mathbf{L}_{\mathbf{3}}$ were grown from the slow evaporation of methanol. $\mathbf{L}_{3}$ crystallised in the triclinic space group $P-1$ and contained one molecule in the asymmetric unit. Like $\mathbf{L}_{\mathbf{1}}$ and $\mathbf{L}_{\mathbf{2}}$ the structure of $\mathbf{L}_{\mathbf{3}}$ is again planar with small mean-plane angles $\left(<11^{\circ}\right)$ between the three main functional groups. The thiourea moiety adopts the anticonformation as per the previous systems. However, unlike $\mathbf{L}_{\mathbf{1}}$ and $\mathbf{L}_{2}$ the imine in $\mathbf{L}_{3}$ adopts a cis-configuration orientating the thiazole nitrogen into a position to be involved in a second intramolecular hydrogen bonding interaction between the second thiourea $\mathrm{NH}$ group and the thiazole nitrogen atom $[\mathrm{N}(3) \cdots \mathrm{N}(1)=$ $2.698(2) \AA$ and $\left.<(\mathrm{N}(3)-\mathrm{H}(3 \mathrm{X}) \cdots \mathrm{N}(1))=136.2(2)^{\circ}\right]$. With both $\mathrm{NH}$ donors involved in intramolecular hydrogen bonding, extension of the structure via H-bonding does not occur, instead there are weak off-set face to face $\pi \cdots \pi$ stacking interactions between the

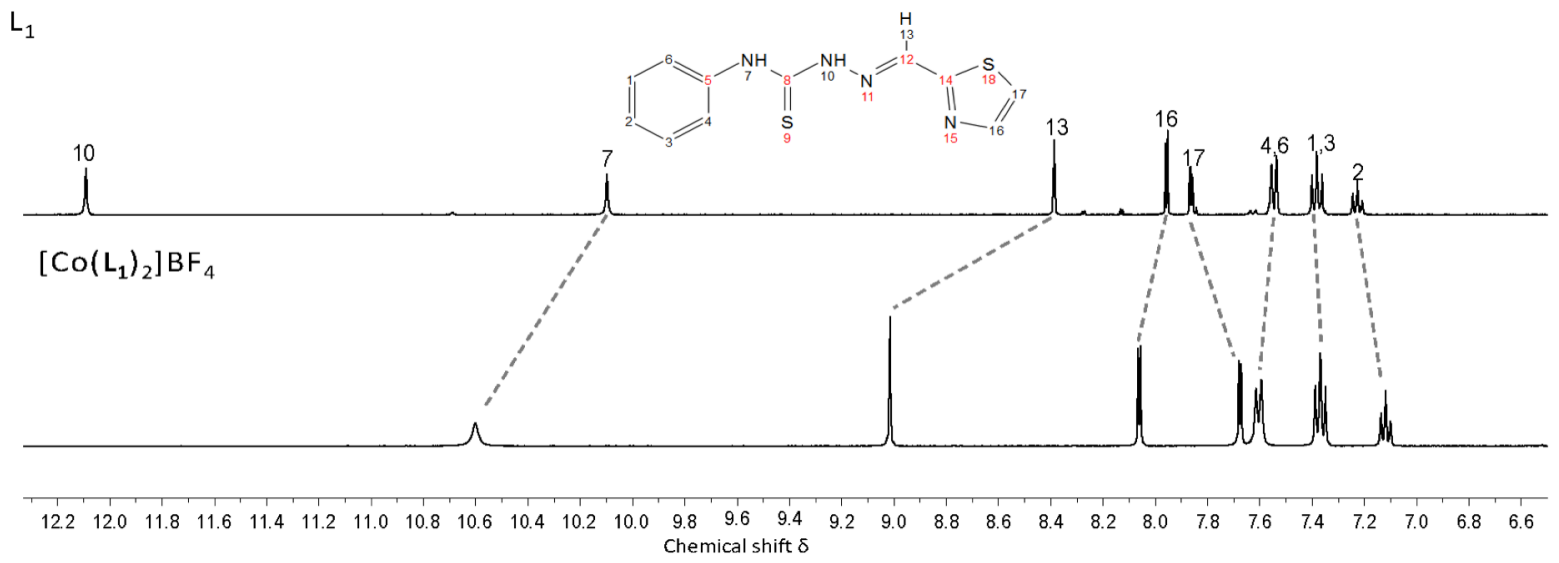

Fig. 3 Stacked plot of ${ }^{1} \mathrm{H}-\mathrm{NMR}$ spectra of $\mathbf{L}_{\mathbf{1}}$ (top) and $\left[\mathrm{Co}\left(\mathbf{L}_{1}\right)_{2}\right]\left(\mathrm{BF}_{4}\right)$ (bottom) recorded in DMSO-d $\mathrm{d}_{6}$ highlighting shifted proton signals on complexation 

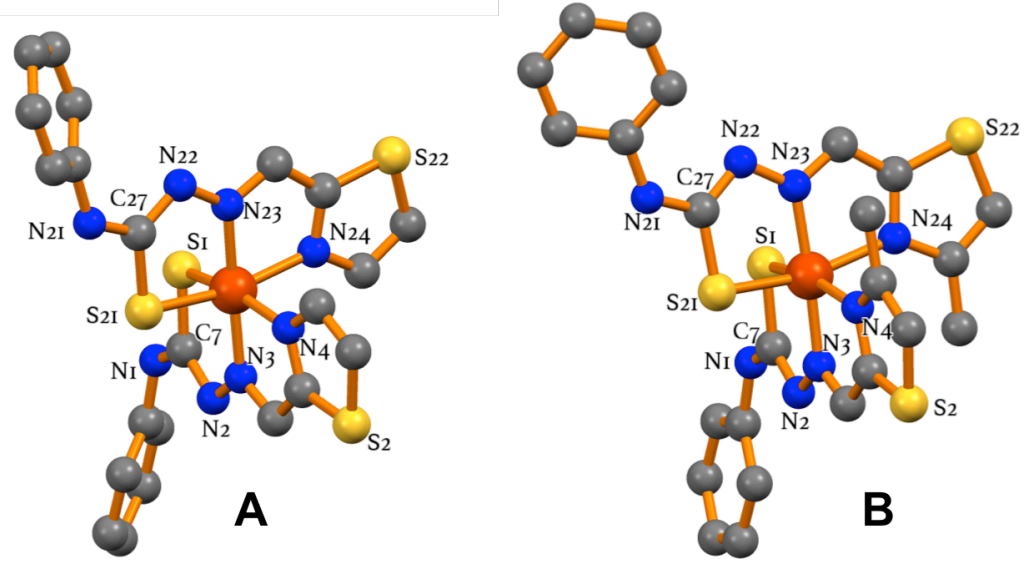

$\begin{array}{cll}\mathrm{L}_{1} & {\left[\mathrm{Fe}\left(\mathrm{L}_{1}\right)_{2}\right] \mathrm{NO}_{3} \cdot \mathrm{H}_{2} \mathrm{O}} & {\left[\mathrm{Co}\left(\mathrm{L}_{1}\right)_{2}\right] \mathrm{BF}_{4} \cdot \mathrm{H}_{2} \mathrm{O}} \\ 1.361(2) & 1.333(3) & 1.334(4) \\ 1.673(1) & 1.742(3) & 1.732(3) \\ - & 1.327(3) & 1.322(4) \\ - & 1.760(2) & 1.744(3) \\ - & 2.220(7) & 2.228(1) \\ - & 2.226(7) & 2.225(1) \\ - & 1.975(2) & 1.945(2) \\ - & 1.931(2) & 1.907(3) \\ - & 1.987(2) & 1.964(3) \\ - & 1.930(2) & 1.902(3) \\ - & 68.7(8) & 53.6(11) \\ - & 80.6-99.8(1) & 81.9-99.2(1)\end{array}$

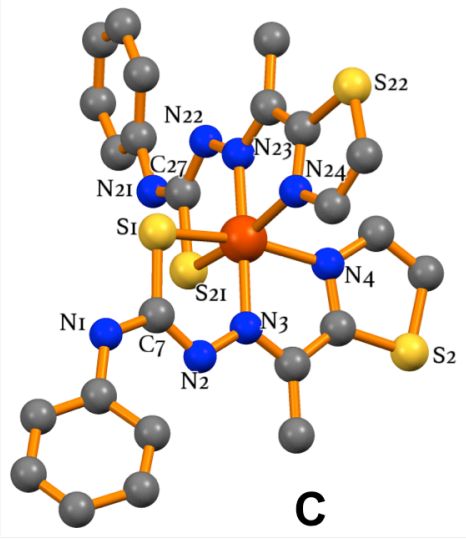

$\mathrm{L}_{2} \quad\left[\mathrm{Fe}\left(\mathrm{L}_{2}\right)_{2}\right] \mathrm{NO}_{3} \cdot \mathrm{H}_{2} \mathrm{O}$

$1.367(5) \quad 1.324(5)$

$1.665(4) \quad 1.764(4)$

$1.330(4)$

$1.751(4)$

$2.220(11)$

$2.226(10)$

$2.019(3)$

$1.928(3)$

$2.034(3)$

$1.924(3)$

$71.2(12)$

81.1 - $101.6(1)$

$\begin{array}{ll}{\left[\mathrm{Co}\left(\mathrm{L}_{2}\right)_{2}\right] \mathrm{BF}_{4} \cdot \mathrm{Et}_{2} \mathrm{O} \mathbf{L}_{3}} \\ 1.321(4) & 1.368 \\ 1.752(3) & 1.677 \\ 1.336(4) & \\ 1.739(3) & \\ 2.2108(9) & \\ 2.2218(9) & \\ 1.978(3) & \\ 1.890(3) & \\ 1.994(3) & \\ 1.890(3) & \\ 49.7(12) & \\ 83.1-100.6(1)\end{array}$

$\left[\mathrm{Fe}\left(\mathrm{L}_{3}\right)_{2}\right] \mathrm{NO}_{3} \cdot 3 / 4 \mathrm{H}_{2} \mathrm{O}$

$1.368(2) \quad 1.294(16)$

$1.677(2) \quad 1.748(12)$

$1.290(14)$

$1.750(12)$

$2.222(3)$

2.207(3)

1.984(10)

$1.925(9)$

$1.966(9)$

$1.916(9)$

$71.6(6)$

80.7 - 104.2 (13)

Fig. 4 Molecular structures (top) of $\left[\mathrm{Fe}\left(\mathbf{L}_{1}\right)_{2}\right]\left(\mathrm{NO}_{3}\right) \cdot \mathrm{H}_{2} \mathrm{O}(\mathrm{A})$, $\left[\mathrm{Fe}\left(\mathbf{L}_{2}\right)_{2}\right]\left(\mathrm{NO}_{3}\right) \cdot \mathrm{H}_{2} \mathrm{O}(\mathrm{B})$, and $\left[\mathrm{Fe}\left(\mathbf{L}_{3}\right)_{2}\right]\left(\mathrm{NO}_{3}\right) \cdot 3 / 4 \mathrm{H}_{2} \mathrm{O}(\mathrm{C})$ showing numbering scheme. Nitrate counter anion, interstitial water molecules and hydrogen atoms are omitted for clarity - see ESI for additional figures of the molecular structures. Table of selected bond lengths and angles (bottom) for all structurally characterised ligands and complexes. $\Sigma$ values for complexes are also tabulated for structurally characterised Fe(III) and Co(III) comnlexes.

thiazole and the phenyl rings on neighbouring molecules [centroid $\cdots$ centroid = 3.74 Å, Fig. S45, ESI].

\subsection{Complex synthesis}

Complexation reactions (Scheme) were carried out in methanol between $\mathbf{L}_{\mathbf{1}}-\mathbf{L}_{3}$ and $\mathrm{Fe}\left(\mathrm{NO}_{3}\right)_{3} \cdot 9 \mathrm{H}_{2} \mathrm{O}$ and $\mathrm{Co}\left(\mathrm{BF}_{4}\right)_{2} \cdot 6 \mathrm{H}_{2} \mathrm{O}$ using a $2: 1$ L:M ratio. In each case the addition of a methanolic solution of metal salt to the methanolic ligand solution resulted in a dramatic colour change from pale yellow to very dark orange/brown. The dark solutions were stirred vigorously whilst open to the air at room temperature for $1 \mathrm{~h}$ before being subjected to vapour diffusion of diethyl ether. In all cases this gave very dark red to orange crystals in reasonable yields (68-80\%). The complexes all adopt the general formula $\left[\mathrm{M}(\mathbf{L})_{2}\right]$ (anion)-solvent with the metal in the +3 oxidation state. The ligand binds through the thioenolate form rather than the thioketo form giving a mono-negative charge on the ligand.

\subsection{Infrared, Mass Spectrometry, NMR and UV/vis spectroscopy}

Infrared spectroscopy provides a valuable tool for analysing these thiosemicarbazone systems. On binding, the ligand undergoes a tautomerism resulting in small spectral changes ( $\mathrm{C}=\mathrm{S}$ to $\mathrm{C}-\mathrm{S}$ ). The comparison between ligands and complexes is shown in Fig S13 S15, ESI. The IR spectra of the complexes also show the expected signals for the $\mathrm{NO}_{3}^{-}$and $\mathrm{BF}_{4}^{-}$anions in the $\mathrm{Fe}(\mathrm{III})$ and $\mathrm{Co}(\mathrm{III})$ complexes respectively. High-resolution mass spectra of all ligands and complexes were collected and showed the expected peaks and isotopic distributions for all ligands and complexes (Fig. S19 - S27, ESI). Electronic spectra of ligands and iron complexes were obtained in $\mathrm{MeOH}$ and in the solid state (Fig S16
- S18, ESI). Spectra of $\mathbf{L}_{\mathbf{1}}-\mathbf{L}_{3}$ (ca. $1 \times 10^{-5} \mathrm{~mol} \mathrm{~L}^{-1}$ in $\mathrm{MeOH}$ ) displayed similar spectral features with intense absorptions at $\lambda_{\max } \sim 350$ $\mathrm{nm}\left(\varepsilon 20,500-22,500 \mathrm{Lmol}^{-1} \mathrm{~cm}^{-1}\right)$. Absorption spectra of Fe(III) complexes ( $c$ a. $1 \times 10^{-5} \mathrm{~mol} \mathrm{~L}^{-1}$ in $\mathrm{MeOH}$ ) showed intense broad charge-transfer absorptions at $\sim 400 \mathrm{~nm}\left(\varepsilon \sim 43,000 \mathrm{Lmol}^{-1} \mathrm{~cm}^{-1}\right)$ accounting for the intense colour of the Fe(III) complexes. The Fe(III) complexes appeared to be stable in solution over long periods of time as the spectra collected some 4 weeks later were unchanged. ${ }^{1} \mathrm{H}-\mathrm{NMR}$ spectra were collected of the three Co(III) complexes in $d_{6}$-DMSO (Fig. S2, S5, S8, ESI). In each case there was a significantly shifted spectrum when compared to the respective ligand spectrum (e.g. the imine protons display a $\sim 0.6$ ppm downfield shift) and loss of one $\mathrm{NH}$ resonance indicating successful complexation (Fig. 3, S3, S6, S9, ESI). Again the complexes exhibited significant solution stability, as spectra collected 4 weeks apart were unchanged. The observed solution stability indicates that these thiosemicarbazone complexes are potentially ideal for development of supramolecular materials and thus should be suitable for a variety of immobilisation techniques where solution processability is required (e.g. gels, Langmuir-Blodgett films and incorporation into polymers).

\subsection{Crystallographic analysis of $\mathrm{Fe}^{3+}$ complexes}

Crystals of $\left[\mathrm{Fe}\left(\mathbf{L}_{1}\right)_{2}\right]\left(\mathrm{NO}_{3}\right) \cdot \mathrm{H}_{2} \mathrm{O}, \quad\left[\mathrm{Fe}\left(\mathbf{L}_{2}\right)_{2}\right]\left(\mathrm{NO}_{3}\right) \cdot \mathrm{H}_{2} \mathrm{O}$, and $\left[\mathrm{Fe}\left(\mathbf{L}_{3}\right)_{2}\right]\left(\mathrm{NO}_{3}\right) \cdot 3 / 4 \mathrm{H}_{2} \mathrm{O}$ were obtained as large red/orange rods or plates by diffusion of diethyl ether into methanolic solutions of the complexes and the low temperature $(100 \mathrm{~K})$ structures obtained (Fig. 4). $\left[\mathrm{Fe}\left(\mathbf{L}_{1}\right)_{2}\right]\left(\mathrm{NO}_{3}\right) \cdot \mathrm{H}_{2} \mathrm{O}$ crystallised in the monoclinic spacegroup $P 2_{1} / \mathrm{c},\left[\mathrm{Fe}\left(\mathbf{L}_{2}\right)_{2}\right]\left(\mathrm{NO}_{3}\right) \cdot \mathrm{H}_{2} \mathrm{O}$ crystallised in the monoclinic space group $P 2_{1} / \mathrm{n}$ and $\left[\mathrm{Fe}\left(\mathbf{L}_{3}\right)_{2}\right]\left(\mathrm{NO}_{3}\right) \cdot 3 / 4 \mathrm{H}_{2} \mathrm{O}$ crystallised in the orthorhombic space group Pna2 ${ }_{1}$. In all cases the asymmetric unit contained one complete molecule and one 

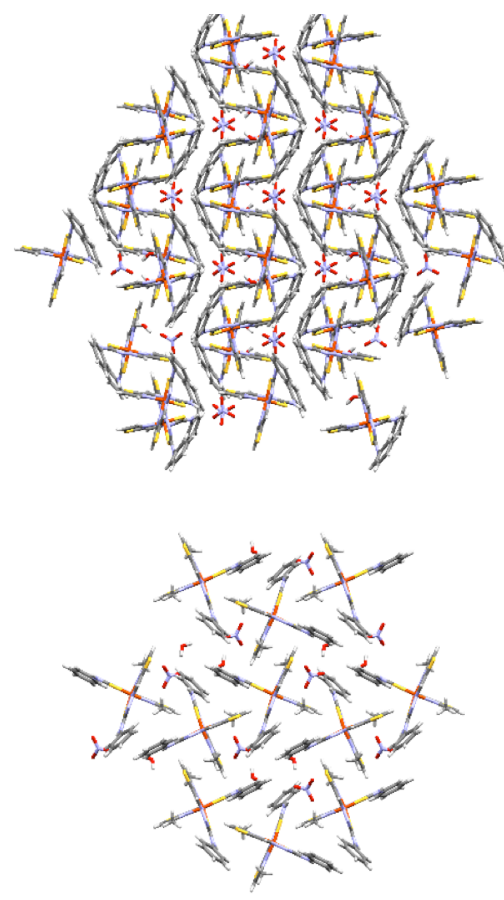

Fig. 5 Packing diagrams of $\left[\mathrm{Fe}\left(\mathbf{L}_{1}\right)_{2}\right]\left(\mathrm{NO}_{3}\right) \cdot \mathrm{H}_{2} \mathrm{O}$ (top) showing spiralled layer formation and $\left[\mathrm{Fe}\left(\mathbf{L}_{2}\right)_{2}\right]\left(\mathrm{NO}_{3}\right) \cdot \mathrm{H}_{2} \mathrm{O}$ showing both the orientation of the packing along the crystallographic c-axis (bottom).

interstitial water molecule (either full or $3 / 4$ occupancy). The general structure of each complex is similar where the Fe(III) centre adopts a distorted octahedral geometry with an $\mathrm{S}_{2} \mathrm{~N}_{4}$ coordination sphere. Bond lengths and angles are consistent with low-spin Fe(III) and are summarised in Figure 4. Analysis of the distortion parameter, $\Sigma$ (sum of the deviation of the cis bond angles from $\left.90^{\circ}\right), \mathbf{1 8 , 2 1 , 4 4 , 4 5}$ indicated relatively small distortion from purely octahedral geometry (ranging from $68.7^{\circ}$ to $71.5^{\circ}$ ). The data also reaffirms the IR spectroscopic interpretation of changes in bond lengths for the $\mathrm{C}=\mathrm{S}$ and $\mathrm{C}-\mathrm{N}$ bonds (Fig. 4). Packing in the solid state is an important area of consideration for potential SCO systems as intermolecular interactions can increase communication between neighbouring molecules and therefore enhance the cooperative nature of the magnetism. In these systems the ligands have many functional groups capable of extending the structure through supramolecular interactions (e.g. H-bonding, $\pi \cdots \pi$ stacking, anion $\cdots \pi$ and $\mathrm{CH} \cdots \pi$ interactions) making analysis of the packing important. $\left[\mathrm{Fe}\left(\mathbf{L}_{1}\right)_{2}\right]\left(\mathrm{NO}_{3}\right) \cdot \mathrm{H}_{2} \mathrm{O}$ displays hydrogen bonding interactions between thioamide $\mathrm{NH}$ groups on neighbouring complexes $(\mathrm{N}(4)$ ' and $\mathrm{N}(24)$ ) and a nitrate counter anion $\left[\mathrm{N}(4)^{\prime} \cdots \mathrm{O}(102)=2.877(3) \AA\right.$ and $<\left(\mathrm{N}(4)^{\prime}-\right.$ $\left.\mathrm{H}(4 \mathrm{X})^{\prime} \cdots \mathrm{O}(102)\right)=172^{\circ} ; \mathrm{N}(24) \cdots \mathrm{O}(102)=2.845(3) \AA$ and $\left.<(\mathrm{N}(24)-\mathrm{H}(24 \mathrm{X}) \cdots \mathrm{O}(102))=160^{\circ}\right]$ where the $\mathrm{NO}_{3}{ }^{-}$acts as a bridge between two complex molecules (Fig S44, ESI). The interstitial water molecule further bridges the nitrate anion to a third molecule by acting as a hydrogen bond donor to a nitrate oxygen atom $(\mathrm{O}(100))$ and the thioenolate nitrogen atom $(\mathrm{N}(23)$ ') on a neighbouring molecule $[\mathrm{O}(200) \cdots \mathrm{O}(100)=2.856(3) \AA$ and $<(\mathrm{O}(200)-\mathrm{H}(20 \mathrm{Y}) \cdots \mathrm{O}(100))=176^{\circ} ; \mathrm{O}(200) \cdots \mathrm{N}(23) "=2.924(3) \AA$ and $\left.<\left(\mathrm{O}(200)-\mathrm{H}(20 \mathrm{Y}) \cdots \mathrm{N}(23)^{\prime \prime}\right)=174^{\circ}\right]$. The $\mathrm{NH}$ and $\mathrm{OH}$ hydrogen bonding interactions described above link neighbouring complexes into a 2D hydrogen bonded network (Fig. 5). Packing in $\left[\mathrm{Fe}\left(\mathbf{L}_{2}\right)_{2}\right]\left(\mathrm{NO}_{3}\right) \cdot \mathrm{H}_{2} \mathrm{O}$ is slightly different in that the nitrate counter anion does not act as a bridge between two thioamide $\mathrm{NH}$ groups on neighbouring complex molecules. Instead the nitrate anion acts as an acceptor in a hydrogen bond to one thioamide $\mathrm{NH}$
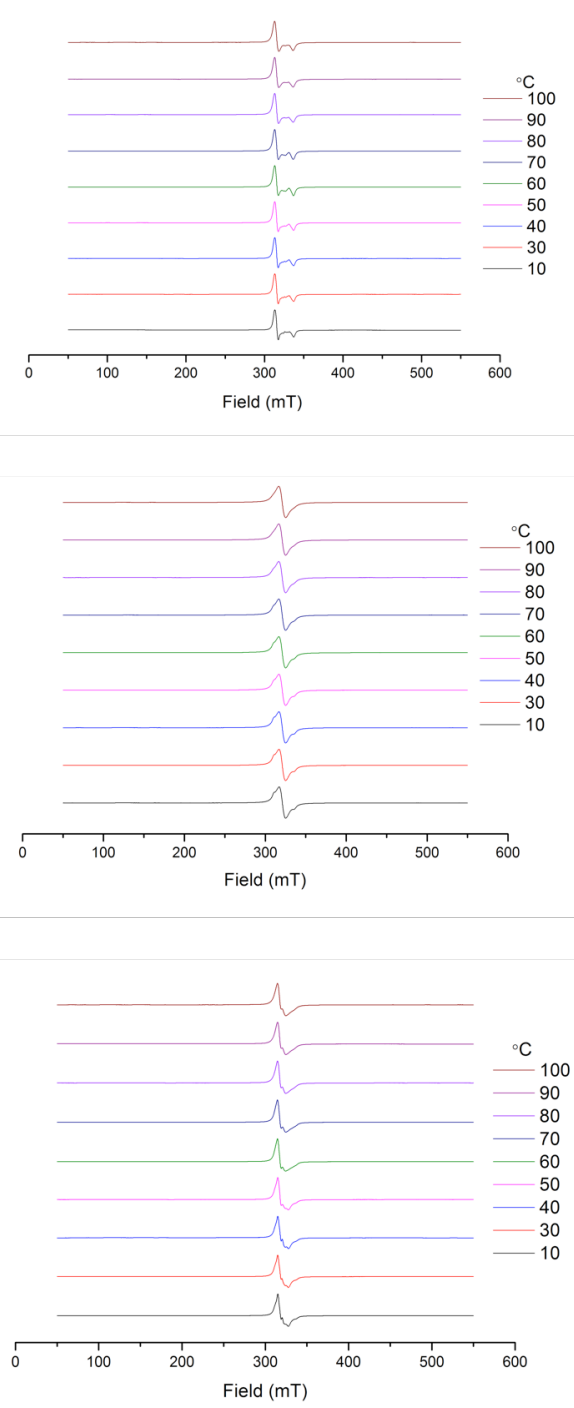

Fig. 6 Variable temperature EPR of $\left[\mathrm{Fe}\left(\mathbf{L}_{1}\right)_{2}\right]\left(\mathrm{NO}_{3}\right) \cdot \mathrm{H}_{2} \mathrm{O}$ (top), $\left[\mathrm{Fe}\left(\mathbf{L}_{2}\right)_{2}\right]\left(\mathrm{NO}_{3}\right) \cdot \mathrm{H}_{2} \mathrm{O}$ (middle) and $\left[\mathrm{Fe}\left(\mathbf{L}_{3}\right)_{2}\right]\left(\mathrm{NO}_{3}\right) \cdot 3 / 4 \mathrm{H}_{2} \mathrm{O}$ (bottom).

$\left[\mathrm{N}(21) \cdots \mathrm{O}(100)=2.822(4) \AA\right.$ and $\left.<(\mathrm{N}(21)-\mathrm{H}(21) \cdots \mathrm{O}(100))=171^{\circ}\right]$ and also to the interstitial water $\left[\mathrm{O}(200) \cdots \mathrm{O}(100)^{\prime}=2.797(5) \AA\right.$ and $\left.<\left(\mathrm{O}(200)-\mathrm{H}(20 \mathrm{~A}) \cdots \mathrm{O}(100)^{\prime}\right)=172^{\circ}\right]$. This in turn acts as the acceptor to the other thioamide $\mathrm{NH}\left[\mathrm{N}(1) \cdots \mathrm{O}(200)^{\prime}=2.885(5) \AA\right.$ and $\left.<(\mathrm{N}(1)-\mathrm{H}(1) \cdots \mathrm{O}(200))=174^{\circ}\right]$. The end result is the linking of molecules through $\mathrm{H}$-bonding interactions to the nitrate and interstitial water molecules giving rise to a complex $\mathrm{H}$-bonding network (Fig. 5). Packing in $\left[\mathrm{Fe}\left(\mathbf{L}_{3}\right)_{2}\right]\left(\mathrm{NO}_{3}\right) \cdot 3 / 4 \mathrm{H}_{2} \mathrm{O}$ is similar to that of $\left[\mathrm{Fe}\left(\mathbf{L}_{2}\right)_{2}\right]\left(\mathrm{NO}_{3}\right) \cdot \mathrm{H}_{2} \mathrm{O}$. Again, the nitrate anion acts as an acceptor in a hydrogen bond to one thioamide $\mathrm{NH}\left[\mathrm{N}(1) \cdots \mathrm{O}(102)^{\prime}=\right.$ $2.929(15) \AA$ and $\left.<\left(\mathrm{N}(1)-\mathrm{H}(1) \cdots \mathrm{O}(102)^{\prime}\right)=151^{\circ}\right]$ and also to the interstitial water $\left[\mathrm{O}(200) \cdots \mathrm{O}(102)^{\prime}=2.888(16) \AA\right.$ and $<(\mathrm{O}(200)$ $\left.\left.\mathrm{H}(20 \mathrm{~B}) \cdots \mathrm{O}(102)^{\prime}\right)=169^{\circ}\right]$. This in turn acts as the acceptor to the other thioamide $\mathrm{NH}[\mathrm{N}(21) \cdots \mathrm{O}(200)=3.008(15) \AA$ and $<(\mathrm{N}(21)$ $\left.\mathrm{H}(21) \cdots \mathrm{O}(200))=172^{\circ}\right]$. Overall, the packing in these Fe(III) systems is complex and extensive, and there are multiple supramolecular connections that link neighbouring molecules within the crystal structure (Fig. S46, ESI).

3.5 Crystallographic analysis of $\mathrm{Co}^{3+}$ complexes

Crystals of $\left[\mathrm{Co}\left(\mathbf{L}_{1}\right)_{2}\right]\left(\mathrm{BF}_{4}\right) \cdot \mathrm{H}_{2} \mathrm{O}, \quad\left[\mathrm{Co}\left(\mathbf{L}_{2}\right)_{2}\right]\left(\mathrm{BF}_{4}\right) \cdot \mathrm{Et}_{2} \mathrm{O}, \quad$ and $\left[\mathrm{Co}\left(\mathbf{L}_{3}\right)_{2}\right]\left(\mathrm{BF}_{4}\right)$ were also obtained as red plate-like crystals by 
diffusion of diethyl ether into methanolic solutions of the complexes and the Low temperature $(100 \mathrm{~K})$ structures studied. $\left[\mathrm{Co}\left(\mathbf{L}_{1}\right)_{2}\right]\left(\mathrm{BF}_{4}\right) \cdot \mathrm{H}_{2} \mathrm{O}$ crystallised in the monoclinic space group $P 2_{1} / \mathrm{n},\left[\mathrm{Co}\left(\mathbf{L}_{2}\right)_{2}\right]\left(\mathrm{BF}_{4}\right) \cdot \mathrm{Et}_{2} \mathrm{O}$ in the monoclinic space group $P 2_{1} / \mathrm{c}$ and $\left[\mathrm{Co}\left(\mathbf{L}_{3}\right)_{2}\right]\left(\mathrm{BF}_{4}\right)$ in the orthorhombic space group Pbca. The general structure of each complex is similar to the Fe(III) systems where the $\mathrm{Co}(\mathrm{III})$ centre adopts a distorted octahedral geometry with a $\mathrm{S}_{2} \mathrm{~N}_{4}$ coordination sphere. In the Co(III) systems the coordination geometries are closer to pure octahedral than the $\mathrm{Fe}(\mathrm{III})$ analogues (distortion parameter $\Sigma$ range $=48.5-53.6^{\circ}$ ) Packing interactions are also somewhat similar to the Fe(III) versions with the exception of $\left[\mathrm{Co}\left(\mathbf{L}_{2}\right)_{2}\right]\left(\mathrm{BF}_{4}\right) \cdot \mathrm{Et}_{2} \mathrm{O}$ which has a disordered partial occupancy $\mathrm{Et}_{2} \mathrm{O}$ molecule within the crystal lattice. $\left[\mathrm{Co}\left(\mathbf{L}_{1}\right)_{2}\right]\left(\mathrm{NO}_{3}\right) \cdot \mathrm{H}_{2} \mathrm{O}$ displays hydrogen bonding interactions between the thioamide $\mathrm{NH}$ groups on neighbouring complexes (N(4)' and $\mathrm{N}(24))$ and a tetrafluoroborate counter anion $\left[\mathrm{N}(4)^{\prime} \cdots \mathrm{F}(4)=2.896(3) \AA \AA\right.$ and $<\left(\mathrm{N}(4)^{\prime}-\mathrm{H}(4 \mathrm{X})^{\prime} \cdots \mathrm{F}(4)\right)=171^{\circ}$; $\mathrm{N}(24) \cdots \mathrm{F}(4)=2.882(4) \quad \AA$ and $\left.<(\mathrm{N}(24)-\mathrm{H}(24 \mathrm{X}) \cdots \mathrm{F}(4))=161^{\circ}\right]$ where the $\mathrm{BF}_{4}^{-}$acts as a bridge between two complex molecules
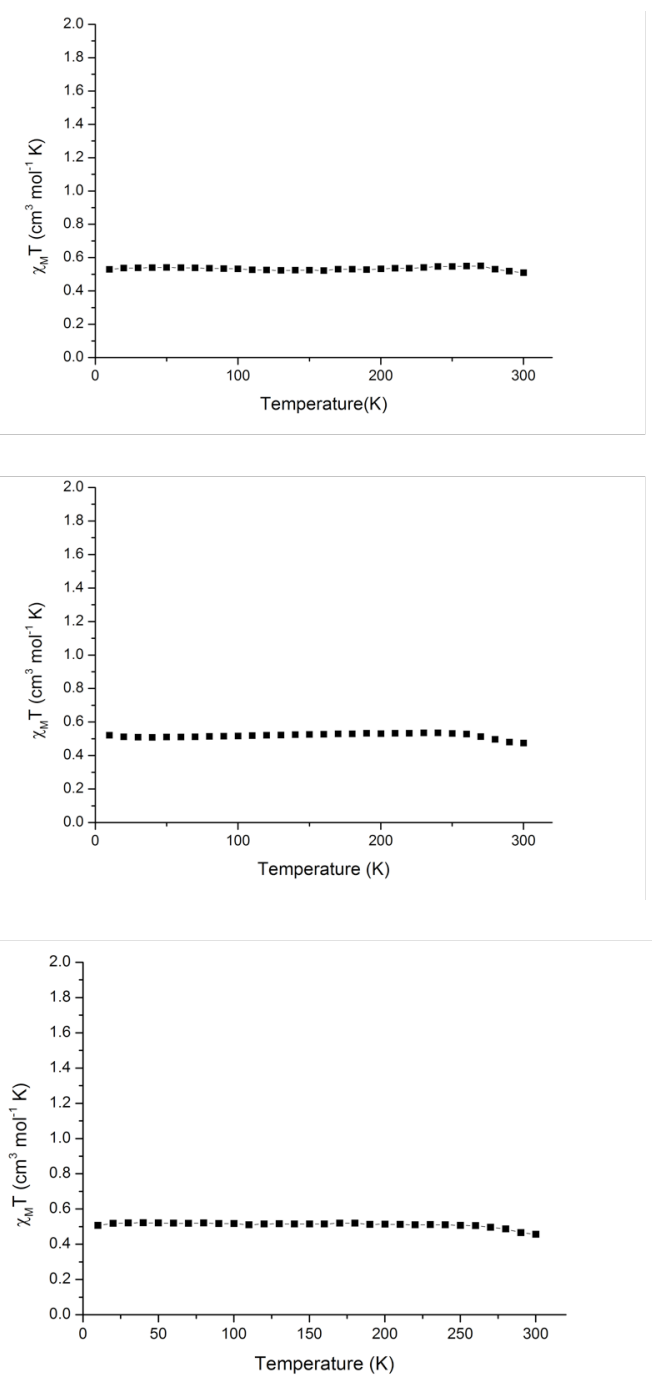

Fig 7 Plots of $\chi_{\mathrm{M}}$ T vs. T for $\left[\mathrm{Fe}\left(\mathbf{L}_{1}\right)_{2}\right]\left(\mathrm{NO}_{3}\right) \cdot \mathrm{H}_{2} \mathrm{O}$ (top), $\left[\mathrm{Fe}\left(\mathbf{L}_{2}\right)_{2}\right]\left(\mathrm{NO}_{3}\right) \cdot \mathrm{H}_{2} \mathrm{O}$ (middle) and $\left[\mathrm{Fe}\left(\mathbf{L}_{3}\right)_{2}\right]\left(\mathrm{NO}_{3}\right) \cdot 3 / 4 \mathrm{H}_{2} \mathrm{O}$ (bottom).

(Fig S41, ESI). The interstitial water molecule further bridges the tetrafluoroborate anion to a third complex molecule by acting as a hydrogen bond donor to two tetrafluoroborate fluorine atoms $(\mathrm{F}(1) \& \mathrm{~F}(2))$ and the thioenolate nitrogen atom $(\mathrm{N}(23)$ ') on a neighbouring molecule $[\mathrm{O}(1) \cdots \mathrm{F}(1)=3.001(5) \AA$ and $<(\mathrm{O}(1)$ $\mathrm{H}(10 \mathrm{Y}) \cdots \mathrm{F}(1))=160^{\circ} ;[\mathrm{O}(1) \cdots \mathrm{F}(2)=3.169(6) \AA$ and $<(\mathrm{O}(1)-$
$\mathrm{H}(10 \mathrm{Y}) \cdots \mathrm{F}(2))=138^{\circ} ; \mathrm{O}(1) \cdots \mathrm{N}(23)^{\prime \prime}=2.861(5) \AA$ and $<(\mathrm{O}(1)-$ $\left.\mathrm{H}(10 \mathrm{X}) \cdots \mathrm{N}(23) ”)=165^{\circ}\right]$. The $\mathrm{NH}$ and $\mathrm{OH}$ hydrogen bonding interactions described above link neighbouring complexes into a 2D hydrogen bonded network. Packing in $\left[\mathrm{Co}\left(\mathbf{L}_{2}\right)_{2}\right]\left(\mathrm{BF}_{4}\right) \cdot \mathrm{H}_{2} \mathrm{O}$ is slightly different in that the tetrafluoroborate counter anion does not act as a bridge between two thioamide $\mathrm{NH}$ groups on neighbouring complex molecules. Instead neighbouring complexes interact directly with each other through the thioamide $\mathrm{NH}$ and an adjacent thioamide $\mathrm{S}\left[\mathrm{N}(24) \cdots \mathrm{S}(2)^{\prime}=\right.$ $3.428(3) \AA$ and $\left.<\left(\mathrm{N}(24)-\mathrm{H}(24 \mathrm{X}) \cdots \mathrm{S}(2)^{\prime}\right)=159^{\circ}\right]$ and the thioamide $\mathrm{NH}$ with the tetrafluoroborate counter anion $[\mathrm{N}(4) \cdots \mathrm{F}(1)=$ $2.883(4) \AA$ and $\left.<(\mathrm{N}(4)-\mathrm{H}(4) \cdots \mathrm{F}(1))=145^{\circ}\right]$.

\subsection{Electron Paramagnetic Resonance and Magnetism}

Variable temperature X-band EPR also confirmed the predominantly low spin nature of these complexes. Measurements on all complexes, between 283 and $373 \mathrm{~K}$, exhibit a g factor of $c a$. 2.1, indicative of low spin Fe(III) complexes (Fig. 6). Variable temperature magnetic susceptibility measurements were obtained for crystalline samples of $\left[\mathrm{Fe}\left(\mathbf{L}_{1}\right)_{2}\right]\left(\mathrm{NO}_{3}\right) \cdot \mathrm{H}_{2} \mathrm{O}$, $\left[\mathrm{Fe}\left(\mathbf{L}_{2}\right)_{2}\right]\left(\mathrm{NO}_{3}\right) \cdot \mathrm{H}_{2} \mathrm{O}$, and $\left[\mathrm{Fe}\left(\mathbf{L}_{3}\right)_{2}\right]\left(\mathrm{NO}_{3}\right) \cdot 3 / 4 \mathrm{H}_{2} \mathrm{O}$ between 10 and $400 \mathrm{~K}$ in an applied field of $1000 \mathrm{Oe}$. All three Fe(III) complexes exhibit very similar properties with a static $\chi_{\mathrm{M}} \mathrm{T}$ value of $0.5 \mathrm{~cm}^{3}$ mol-1 $^{-1}$ between 10 and $300 \mathrm{~K}$ (Fig. 7). The $S=1 / 2$ low spin assignment at low temperatures is in accordance with the bond lengths of approximately $2 \AA$ for the Fe-N donors.

\section{Conclusions}

We have reported on the synthesis and characterisation of six new Fe(III) and Co(III) complexes prepared from novel thiosemicarbazone ligands. Variable temperature magnetic measurements and EPR spectra of the Fe(III) systems showed that they adopted a low spin configuration from 10 $300 \mathrm{~K}$. The complexes displayed significant solution stability, as assessed through electronic spectra for Fe(III) complexes and NMR spectra for Co(III) complexes run four weeks apart. The solid-state structures displayed many intermolecular interactions linking the complexes into intricate arrays. The combination of significant structure extension through supramolecular interactions and solution stability is ideal for the development of supramolecular materials as the systems should be suitable for a variety of immobilisation techniques where solution processability is required (e.g. gels, Langmuir-Blodgett films and incorporation into polymers). We are currently investigating the development of such systems through incorporation of structure directing substituents into the ligands. Additionally, the ligand family presented herein is similar to known SCO ligands, thus it represents an upper limit to ligand field strength, laying the foundation for tailored design of these ligands for highly processable solution-stable SCO species. Addition of electron-withdrawing groups to the thiazole ring is expected to favourably alter the ligand field strength.

\section{Acknowledgements}

The authors are grateful to the University of Southampton and University College Dublin for support of this work. JAK thanks the EPSRC for funding through grant references 
EP/K039466/1 and EP/K014382/1. ABC thanks the Leverhulme Trust for the award of a Study Abroad Studentship (SAS2016-38). GGM would like to thank Science Foundation Ireland for Investigator Project Award 12/IP/1703, The National University of Ireland and the Cultural Service of the French Embassy in Ireland for scholarships (to A.J.F) and the Irish Higher Education Authority for funding for a SQUID magnetometer.

\section{References}

1 J. P. Byrne, J. A. Kitchen and T. Gunnlaugsson, Chem. Soc. Rev. Chem., 2014, 43, 5302-5325.

2 J. E. M. Lewis, E. L. Gavey, S. A. Cameron and J. D. Crowley, Chem. Sci., 2012, 3, 778-784.

3 D. J. Wales and J. A. Kitchen, Chem. Cent. J., 2016, 10, 72.

4 A. Ferguson, R. W. Staniland, C. M. Fitchett, M. A. Squire, B. E. Williamson and P. E. Kruger, Dalton Trans., 2014, 43, 1455014553.

5 S. Shanmugaraja, C. Dabadie, K. Byrne, A. J. Savyasachi, D. Umadevi, W. Schmitt, J. A. Kitchen and T. Gunnlaugsson, Chem. Sci., 2017, 8, 1535-1546.

6 C. Bartual-Murgui, A. Akou, C. Thibault, G. Molnár, C. Vieu, L. Salmon and A. Bousseksou, J. Mater. Chem. C, 2015, 3, 12771285.

7 S. Ji, W. Wu, W. Wu, P. Song, K. Han, Z. Wang, S. Liu, H. Guo and J. Zhao, J. Mater. Chem., 2010, 20, 1953-1963.

8 V. W-W. Yam and K. M-C. Wong, Chem. Commun., 2011, 47, 11579-11592.

9 M. Galli, J. E. M. Lewis and S. M. Goldup, Angew. Chem. Int. Ed., 2015, 54, 13545-13549.

10 D. J. Harding, W. Phonsri, P. Harding, I. A. Gass, K. S. Murray, B. Moubaraki, J. D. Cashion, L. Liu and S. G. Telfer, Chem. Commun., 2013, 49, 6340-6342.

11 M. J. Murphy, T. D. Keene, J. R. Price, D. M. D’Alessandro and C. J. Kepert, Aust. J. Chem., 2014, 67, 1607-1611.

12 R. E. Powell, C. H. Schwalbe, G. J. Tizzard and P. J. van Koningsbruggen, Acta Crystallogr. Sect. C Struct. Chem., 2014, 70, 595-598.

13 S. Floquet, E. Rivière, K. Boukheddaden, D. Morineau and M.-L. Boillot, Polyhedron, 2014, 80, 60-68.

14 Z.-Y. Li, J.-W. Dai, Y. Shiota, K. Yoshizawa, S. Kanegawa and O. Sato, Chem. Eur. J., 2013, 19, 12948-12952.

15 Z.-Y. Li, J.-W. Dai, K. J. Gagnon, H.-L. Cai, T. Yamamoto, Y. Einaga, H.-H. Zhao, S. Kanegawa, O. Sato, K. R. Dunbar and R.-G. Xiong, Dalton Trans., 2013, 42, 14685-14688.

16 E. W. Y. Tido, C. Faulmann, R. Roswanda, A. Meetsma and P. J. van Koningsbruggen, Dalton Trans., 2010, 39, 1643-1651.

17 S. Floquet, M. C. Muñoz, R. Guillot, E. Rivière, G. Blain, J.-A. Réal and M.-L. Boillot, Inorg. Chim. Acta, 2009, 362, 56-64.

18 J. A. Kitchen, N. G. White, G. N. L. Jameson, J. L. Tallon and S. Brooker, Inorg. Chem., 2011, 50, 4586-4597.
19 J. A. Kitchen, A. Noble, C. D. Brandt, B. Moubaraki, K. S. Murray and S. Brooker, Inorg. Chem., 2008, 47, 9450-9458.

20 J. A. Kitchen, N. G. White, M. Boyd, B. Moubaraki, K. S. Murray, P. D. W. Boyd and S. Brooker, Inorg. Chem., 2009, 48, 66706679.

21 J. A. Kitchen, J. Olguín, R. Kulmaczewski, N. G. White, V. A. Milway, G. N. L. Jameson, J. L. Tallon and S. Brooker, Inorg. Chem., 2013, 52, 11185-11199.

22 J. A. Kitchen, G. N. L. Jameson, J. L. Tallon and S. Brooker, Chem. Commun., 2010, 46, 3200-3202.

23 N. G. White, H. L. C. Feltham, C. Gandolfi, M. Albrecht and S. Brooker, Dalton Trans., 2010, 39, 3751-3758.

24 M. Cavallini, Phys. Chem. Chem. Phys., 2012, 14, 11867-11876.

25 J. A. Kitchen, N. Zhang, A. B. Carter, A. J. Fitzpatrick and G. G. Morgan, J. Coord. Chem., 2016, 69, 2024-2037.

26 A. J. Fitzpatrick, P. N. Martinho, B. J. Gildea, J. D. Holbrey and G. G. Morgan, Eur. J. Inorg. Chem., 2016, 2025-2029.

27 P. N. Martinho, C. J. Harding, H. Müller-Bunz, M. Albrecht and G. G. Morgan, Eur. J. Inorg. Chem., 2010, 675-679.

28 E. N. W. Howe, N. Busschaert, X. Wu, S. N. Berry, J. Ho, M. E. Light, D. D. Czech, H. A. Klein, J. A. Kitchen and P. A. Gale, J. Am. Chem. Soc., 2016, 138, 8301-8308.

29 B. Ghosh, P. Adak, S. Naskar, B. Pakhira, P. Mitra, R. Dinda, S. K. Chattopadhyay, Inorg. Chim. Acta., 2017, 459, 1-14.

30 N. A. Ryabova, V. I. Ponomarev, V. V. Zelentsov, V. I. Shipilov and L. O. Atovmyan, J. Struct. Chem., 1981, 22, 234-238.

31 J. R. Dilworth and R. Hueting, Inorg. Chim. Acta., 2012, 389, 315.

32 N. C. Saha, A. Saha, R. J. Butcher, S. Chaudhuri and N. Saha, Inorg. Chim. Acta, 2002, 339, 348-354.

33 M. M. M. Raposo, B. García-Acosta, T. Ábalos, P. Calero, R. Martínez-Máñez, J. V. Ros-Lis and J. Soto, J. Org. Chem., 2010, 75, 2922-2933.

34 R. Venkatraman, H. Ameera, L. Sitole, E. Ellis, F. R. Fronczek and E. J. Valente, J. Chem. Crystallogr., 2009, 39, 711-718.

35 R. Venkatraman, M. A. Hossain and F. R. Fronczek, Acta Crystallogr. Sect. E Struct. Rep. Online, 2010, 66, 541-542.

36 R. Venkatraman, D. S. Samuel, Z. Arslan, M. A. Hossain and F. R. Fronczek, Acta Crystallogr. Sect. E Struct. Rep. Online, 2013, 69, 246-247.

37 S. R. Arumugam, S. S. R. Dasary, R. Venkatraman, H. Yu and F. R. Fronczek, Acta Crystallogr. Sect. E Struct. Rep. Online, 2011, 67, 1409-1410.

38 S. S. R. Dasary, S. R. Arumugam, H. Yu, R. Venkatraman and F. R. Fronczek, Acta Crystallogr. Sect. E Struct. Rep. Online, 2011, 67, 816-817.

39 S. J. Coles and P. A. Gale, Chem. Sci., 2012, 3, 683-689.

40 CrystalClear-SM Expert 3.1, (Rigaku, 2012).

41 CrysAlisPro 38.41, (Rigaku Oxford Diffraction, 2015).

42 G. M. Sheldrick, Acta Crystallogr. A, 2008, 64, 112-122.

43 O. V. Dolomanov, L. J. Bourhis, R. J. Gildea, J. A. K. Howard and

H. Puschmann, J. Appl. Crystallogr., 2009, 42, 339-341.

44 R. G. Miller and S. Brooker, Inorg. Chem., 2015, 54, 5398-5409.

45 J. Nelson, V. McKee, G. Morgan, Prog. Inorg. Chem. 1998, 47, 167-317 